\title{
Patient and family engagement in Alberta's Strategic Clinical Networks
}

\author{
Mikie Mork MSc BPE, Garry Laxdal DBA, Gloria Wilkinson RN
}

\author{
— Cite as: CMAJ 2019 December 4;191(Suppl 1):S4-6. doi: 10.1503/cmaj.190596
}

$\mathbf{T}$ he Patient First Strategy of Alberta Health Services (AHS) ${ }^{1}$ mandates that listening to patients, families and caregivers and valuing their input is essential to develop solutions that support health and well-being in the province. ${ }^{2}$ Evidence suggests that patient engagement can lead to better health outcomes, contribute to improvement in quality and patient safety, and help control costs of health care. ${ }^{3}$ As such, Alberta's 16 Strategic Clinical Networks (SCNs) have embraced patient engagement as a foundational principle for establishing networks: about 150 patient and family advisors have been embedded within the networks. These volunteer advisors are seen as experts grounded in the patient experience; they serve as educators and mentors to other patients and families; and they represent all communities, ages, genders, cultures and geographical locations.

Each SCN supports patients, families and caregivers to share their experiences and passion to make positive change. Patient and family advisors and patient and community engagement researchers (PaCERs), who bring together their personal health experiences with specialized training and conduct research ${ }^{2}$ within Alberta's SCNs, are active and valuable partners at all levels and in all associated activities of the SCNs. This includes being members of leadership teams, core committees, interview panels and working groups. Patient and family advisors and PaCERs are partners in policy development, priority and agenda setting and health system research and innovation, and speakers at relevant local and provincial meetings. The patient voice is unique, and those with first-hand experience provide important insights when setting priorities and exploring solutions toward improved outcomes.

The SCNs are establishing an understanding of how patient and family engagement can improve the health system. They are tracking retention rates of advisors by using a volunteer database with start and end dates, and are now in the second year of collecting data through an annual patient engagement survey to assist with understanding what is going well and where improvements can be made. Analyses of these data will inform strategies to maximize retention of patient and family advisors.

Several successful initiatives have been co-designed that serve as exemplars in patient engagement. To ensure that active patient and family advisors can function effectively in their advi-

\section{KEY POINTS}

- A growing body of evidence suggests that patient engagement can lead to better health outcomes, contribute to improvement in quality of care and patient safety, and help control health care costs.

- Alberta's 16 Strategic Clinical Networks (SCNs) have embraced meaningful patient engagement as a foundational principle for establishing networks, and, to that end, they have embedded about 150 patient and family advisors within the networks.

- The SCNs are establishing an understanding of the effect of patient and family engagement to make improvements in the health system; several successful initiatives that are exemplars in meaningful engagement have been designed.

sory role, they are supported by SCN staff liaisons as their main point of contact. Engaging for Excellence: A Staff Liaison's Guide to Best Practice ${ }^{4}$ was co-designed by the advisors and SCN staff. Written from the patient advisors' perspective, the guide shares tips on meaningful engagement related to maintaining mutual respect, timing of engagement, applying co-design principles in project work, techniques that help to level the playing field (e.g., avoid titles, jargon, acronyms and labels), the importance of checking in with people who are quiet, and ensuring that expenses are reimbursed promptly (Appendix 1, available at www.cmaj.ca/lookup/suppl/doi:10.1503/cmaj.190596/-/DC1).4,5

For some, the Patient Engagement Reference Group (see photo) provides a meeting forum for patient and family advisors and PaCERs in the SCN to develop trust in a safe environment, and to share experiences and learn from each other. Advisors connect and network with other advisors, endeavour to understand barriers and challenges in an effort to break down silos regarding patient and family engagement in the SCNs, develop and strengthen skills, and participate in consultations regarding SCN or AHS work. The Patient Engagement Reference Group meetings, which occur 3-4 times a year, combine face-to-face and videoconferencing approaches. They are chaired by an elected patient and family advisor and the SCN chief program officer. These co-chairs have shared responsibilities and decision-making, consistent with best practice as identified in other health care organizations and 
jurisdictions. ${ }^{3,5,6}$ Several members of the reference group have developed digital stories (www.youtube.com/playlist?list=PLi1 tOF1I5ZoWY3hfrrwu15NTWz8e9amOt) that are used across Alberta at many different forums to emphasize patient- and family-centred care. The reference group also helps to inform quality improvement and patient safety, and development of clinical pathways, policies and strategies (e.g., AHS' virtual health strategy). The members consistently self-evaluate; past evaluations strongly indicated that advisors find the meeting opportunities valuable and inspiring, which motivates them in their partnership roles with the SCNs.

In its quest for sustainable and effective care, the PaCER unit at the O'Brien Institute for Public Health (University of Calgary, Calgary) is working to transform the role of patients in health and health care delivery. The PaCERs are patient researchers with a variety of health conditions and trained in qualitative health research, who are creating a new collective research voice by patients, with patients and for patients. Those who graduate from the research program have an informed voice in health systems research, the knowledge to ask the right questions with the right approach, and the ability to go beyond advocacy to be an informed advisor. Working in collaboration with medical researchers, health systems and community organizations, the PaCER unit is committed to finding better, stronger and more creative ways of engaging patients in health care. This means building new partnerships and roles for patients. The PaCERs have published many articles in a range of topics that include acute and intensive care settings, osteoarthritis management, advanced care planning and enhanced recovery after surgery. Involving patients and those who care about them adds a new dimension to traditional research. Sixty-one people have graduated from the PaCERs program since its inception in 2013 and, to date, about 20 are currently engaged in research initiatives with the SCNs. The original intention in developing the PaCER program was to build capacity among those involved with the SCNs. ${ }^{7}$

Healthcare 101 was launched in May 2018 and was codesigned with Albertans for Albertans, aiming to help them experience health care in a more positive way. Patient and family advisors and partner organizations involved with the SCNs together found 4 topics for discussion that they felt patients and family would benefit from: understanding the health care system, navigation of the system, how to talk to doctors and other health care providers, and how to understand their rights. Four free online modules - Healthcare Basics for Albertans, Finding My Way, Being My Own Advocate and My Rights - provide information and tools designed to educate Albertans on a range of topics in health care. ${ }^{8}$ Since its launch in May 2018, Healthcare 101 pages have been viewed more than 11000 times and have been translated into French and Spanish by users. ${ }^{8,9}$

Patient and family engagement is a relatively new area of focus in health system improvement; as learning networks, the SCNs improve through trial and error, failures and successes. Engaging patients is difficult because it is new, and some clinicians and leaders are inexperienced. Through the SCNs' commitment to meaningful engagement, effective learning strategies have been developed and documented in the Engaging for Excellence guide for best practice, which is used to train SCN staff.

By working and learning together, and ensuring the presence of advisors' voices, the SCNs believe that they have developed well-rounded solutions to future challenges. Challenges that the SCNs initially encountered in 2012 included the need to orient everyone to the importance of meaningful patient

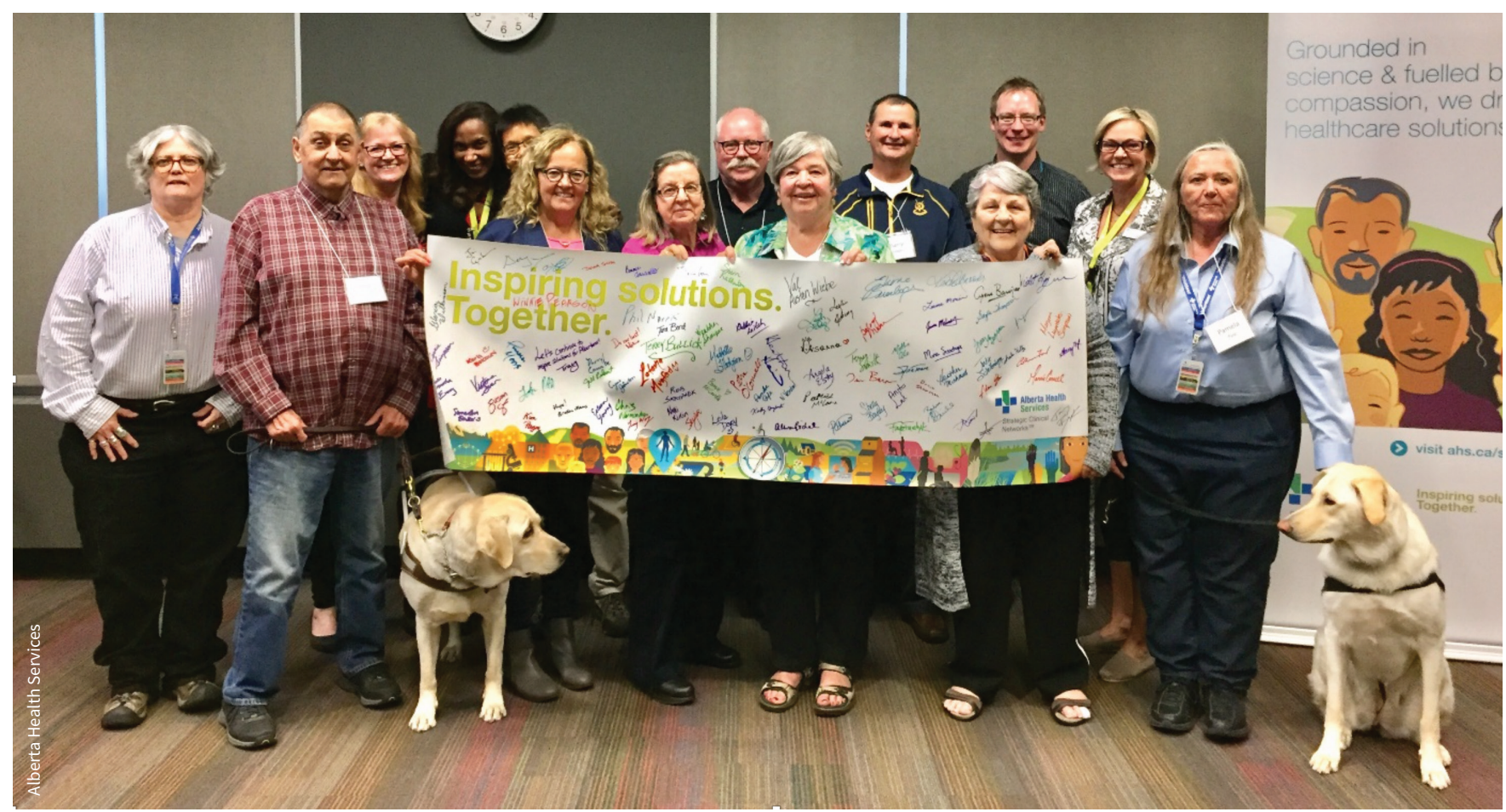

Members of the Strategic Clinical Network Patient Engagement Reference Group. 
engagement without tokenism ${ }^{10}$ and regardless of participants' formal training in health care or traditional hierarchies. The SCNs overcame some of these challenges by embracing codesign principles (which often takes longer but improves the quality of outputs), engaging early in new initiatives to ensure that the patient or family members' voices are heard from start to finish and, where possible, ensuring that there is more than 1 Patient and Family Advisor engaged in any given initiative for ongoing support. The SCNs have adopted the philosophy, "we are all students of engagement," and continue to learn and grow together.

By continuing to provide meaningful engagement opportunities, education, developing the Patient Engagement Reference Group and leading by example, the SCNs have been able to successfully recruit and retain advisors. Ongoing endeavours to learn and follow best practice will help to overcome challenges to patient engagement within the SCNs. They are committed to strengthening the relationships with patient and family advisors, engaging them as valuable partners in decision-making, supporting them as they make important contributions, and prioritizing work that improves health outcomes and the experiences of patients and their families. Ongoing evaluations of these exemplars are either in progress or have been completed using formal surveys or focus groups.

\section{References}

1. Patient First Strategy. Edmonton: Alberta Health Services. Available: www. albertahealthservices.ca/info/Page11981.aspx (accessed 2019 May 15).

2. Strilchuk A, Mork M, Furdyk F. Alberta's Strategic Clinical Networks Roadmap 2019-2024. Calgary: Alberta Health Services; 2019. Available: www.alberta healthservices.ca/assets/about/scn/ahs-scn-reports-past-future-present.pdf (accessed 2019 Apr. 3).

3. Carman KL, Dardess P, Maurer M, et al. Patient and family engagement: a framework for understadning the elements and developing interventions and policies. Health Aff (Millwood) 2013;32:223-31.

4. Laxdal G, McMeekin S, Wong T, et al. Engaging for excellence: a staff liaison's guide to best practice. Edmonton: Alberta Health Services; 2019. Available: www.albertahealthservices.ca/assets/about/scn/ahs-scn-perg-staff-liaisons -guide-to-best-practice.pdf (accessed 2019 Oct. 24).

5. Witteman HO, Chipenda Dansokho S, Colquhoun H, et al. Twelve lessons learned for effective research partnerships between patients, caregivers, clinicians, academic researchers, and other stakeholders. J Gen Intern Med 2018; 33:558-62.

6. Strategy for Patient-Oriented Research - Patient Engagement Framework. Ottawa: Canadian Institutes of Health Research; modified 2019 May 27. Available: www.cihr-irsc.gc.ca/e/48413.html (accessed 2019 Oct. 24).

7. PaCER - Patient and Community Engagement Research. Calgary: University of Calgary; 2019. Available: https://obrieniph.ucalgary.ca/pacer (accessed 2019 May 15).

8. IMAGINE CITIZENS Collaborating for Health [main page]. Available: https:// imaginecitizens.ca/ (accessed 2019 May 15).

9. Healthcare 101: Co-designed with Albertans, for Albertans. Edmonton: Government of Alberta; 2018. Available: https://myhealth.alberta.ca/HealthTopics/ Healthcare101 (accessed 2019 May 15).

10. Domecq JP, Prutsky G, Elraiyah T, et al. Patient engagement in research: a systematic review. BMC Health Serv Res 2014;14:89.
Competing interests: Mikie Mork is an employee of Alberta Health Services. No other competing interests were declared.

This article has been peer reviewed.

Affiliation: Strategic Clinical Networks, Alberta Health Services, Edmonton, Alta.

Contributors: All of the authors contributed to the conception of the work, drafted the manuscript, revised it critically for important intellectual content, gave final approval of the version to be published and agreed to be accountable for all aspects of the work.

Acknowledgements: The authors would like to express appreciation to the following: Strategic Clinical Network (SCN) patient and family advisors; members of the Patient Engagement Reference Group; SCN Patient Engagement team; Tracy Wasylak; Sean Bagshaw; IMAGINE CITIZENS Collaborating for Health; Health
Quality Council of Alberta; Maria J. Santana (Patient Engagement Platform - Strategy for Patient Oriented Research (SPOR), W21C Research and Innovation Centre, Cumming School of Medicine, University of Calgary, Calgary); Community Engagement Research, O'Brien Institute for Public Health, University of Calgary; and Action Dignity Society, Calgary.

Correspondence to: Mikie Mork, mikie.mork @ahs.ca 